\title{
Extracting fetal heart signal from noisy maternal ECG by multivariate singular spectrum analysis
}

\author{
Mansoureh Ghodsi, Hossein Hassani* and Saeid Sanei
}

Fetal heart rate and its beat-to-beat variability are two important indications about the health and condition of the fetus. The observed maternal electrocardiogram (ECG) signal consists of mother heart signal and fetal heart signal and is often very noisy. In this paper, we propose a multivariate singular spectrum analysis (MSSA) for extracting and separating the mother heart signal, the fetal heart signal and the noise component from the combined ECGs. The proposed method is designed to cope with noisy recordings, which is an important limitation of several approaches proposed in the literature. To validate the proposed algorithm some noisy simulated signals are used. The performance of the technique is also examined using real-life signals.

AMS 2000 SUBJECT CLASSIFICATIONS: 92C55, 94A12. KeYwords AND PHRASES: Singular Spectrum Analysis, Fetal, Maternal, ECG, Noise, Multivariate.

\section{INTRODUCTION}

Fetal heart rate (FHR) and its beat-to-beat variability are two important indications about the health and condition of the fetus [1]. The fetal electrocardiogram (FECG) therefore represents the information about the fetal heart rate and the fetal condition. For example, having information about the fetal heart rate, any arrhythmia or changes in the fetal heart rate can be detected. Moreover, the cardiac waveform contains important diagnostic information. For example, the position and the sign of each part in the ECG waveform is very important to discriminate between various forms of supraventricular tachycardia.

Fetal heart rate monitoring is an important methodology that can provide early information about fetal wellbeing and diagnose those at risk of diseases for possible abnormalities such as sudden infant death syndrome [2] and supraventricular extrasystole [3]. Although the Doppler ultrasound imaging system is currently used for fetal heart rate monitoring, it is not suitable for long term monitoring due to its sensitivity to movement and accessibility limitations [4].

It should be noted that achieving a clear abdominally recorded fetal signal is a very difficult task since when measuring the antepartum FECG from electrodes on the

*Corresponding author.

mother's skin, the much stronger maternal electrocardiogram (MECG) forms the largest source of interference. Furthermore, additional factors like a malnourished fetus, an obese mother, and other noise sources become relatively important in comparison with the low voltage range of the FECG. Note that extracting the antepartum FECG from electrodes on the mother's skin, is performed by the much stronger omnipresent maternal MECG. Therefore, one needs to remove the MECG from the recorded signals before further analysis on the FECG. Note also that, in relatively late stages of pregnancy, including labor, the fetal heart rate can be determined much easier than in earlier stages of pregnancy, when the fetal ECG is much weaker.

Various research has been devoted to FECG extraction; the technique based on singular value decomposition $[5,6]$, cross-correlation techniques [7], adaptive filtering [8, 9], decomposition into orthogonal basis [10], fractals [11], neural networks [12], adaptive filtering combined with genetic algorithms [13], fuzzy logic [14], frequency based technique [15], real-time signal processing [16], independent component analysis for blind source separation [17, 18], waveletbased techniques [19-21], and principal component analysis and projective filtering [22] and references therein, have been very common. However, some of the above mentioned research has several major drawbacks.

Here we consider an alternative method for extracting of the FECG signal. In the proposed method we separate FECG, MECG and noise components using multivariate SSA (MSSA). Since considerable dependence exists among different ECG signals, application of MSSA to multi-lead ECGs can benefit from such an interdependence information. This can not be exploited when signal channel information is processed.

SSA is a powerful technique for time series analysis and signal processing incorporating the elements of classical time series analysis, linear algebra, multivariate statistics, multivariate geometry, dynamical systems and signal processing [23]. This approach circumvents many limitations such as nonlinearity and nonstationarity of the signals. Moreover, contrary to the traditional methods of time series analysis and signal processing, the SSA method is non-parametric and does not require any prior assumption about the data. Furthermore, SSA decomposes a series into its component parts, while excluding the random (noise) component which is very important for the FECG extraction. 
One of the main features of the proposed technique is that the extraction of FECG signal is achieved through successive extraction (or filtering) in an algebraically orthogonal projection.

It is worth mentioning that most of the current algorithms do not consider any potential information hidden in the signal structure. The motivation for this is that by using additional information such as the temporal dynamics and linear and nonlinear interdependency among signals (which we use in SSA, particulary in MSSA), we can improve the performance of existing signal processing methods (see, for example, [24] and [25]).

The rest of the paper is organized as follows: Section 2 describes the SSA technique; the structure of the MECG and FECG signals are considered in Section 3; the proposed algorithm for foetal ECG extraction using MSSA is described in Section 4; the empirical results using the simulated and real data are represented in Section 5; finally, Section 6 is devoted to conclusions.

\section{SINGULAR SPECTRUM ANALYSIS}

The basic SSA method consists of two complementary stages: decomposition and reconstruction; both stages include two separate steps. In the first stage we decompose the series and in the second stage we reconstruct the original series and use the reconstructed series for further analysis. The method has several essential extensions. First, the multivariate version of the method permits simultaneous expansion of several time series; see, for example [26]. Second, the SSA ideas lead to several forecasting procedures for time series; see [23, 26]. Also, the same ideas are used in [23] and [27] for change-point detection in time series. For comparison with classical methods, see [28-31]. For automatic methods of identification within the SSA framework see [32] and for recent work in 'Caterpillar'-SSA software as well as new developments see [33]. A family of causality tests based on the SSA technique has also been considered in [25]. In the area of nonlinear time series analysis, SSA was considered as a technique that could compete with more standard methods. In a number of papers, SSA is considered as a filtering method (see, for example, [34] and references therein). In other research, the noise information extracted using the SSA technique, has been used as a medical diagnostic test [35]. The SSA technique has also been used as a filtering method for longitudinal measurements. It has been shown that noise reduction is important for curve fitting in growth curve models, and that SSA can be employed as a powerful tool for noise reduction for longitudinal measurements [36]. A short description of the SSA technique is given as follows (for more information see [23]).

\subsection{Decomposition}

\section{1st step: Embedding}

Embedding can be regarded as a mapping operation that transfers a one-dimensional time series $Y_{T}=\left(y_{1}, \ldots, y_{T}\right)$ into the multidimensional series $X_{1}, \ldots, X_{K}$ with vectors $X_{i}=\left(y_{i}, \ldots, y_{i+L-1}\right)^{T} \in \mathbf{R}^{L}$, where $K=T-L+1$. The single parameter of the embedding is the window length $L$, an integer such that $2 \leq L \leq T$. The result of this step is the trajectory matrix $\mathbf{X}=\left[\bar{X}_{1}, \ldots, X_{K}\right]=\left(x_{i j}\right)_{i, j=1}^{L, K}$.

\section{2nd step: Singular value decomposition}

The second step, the SVD step, makes the singular value decomposition of the trajectory matrix and represents it as a sum of rank-one bi-orthogonal elementary matrices. Denote by $\lambda_{1}, \ldots, \lambda_{L}$ the eigenvalues of $\mathbf{X X}^{T}$ in decreasing order of magnitude $\left(\lambda_{1} \geq \cdots \geq \lambda_{L} \geq 0\right)$ and by $U_{1}, \ldots, U_{L}$ the orthonormal system of the eigenvectors of the matrix $\mathbf{X X}^{T}$ corresponding to these eigenvalues. Set $d=\max \left(i\right.$, such that $\left.\lambda_{i}>0\right)=\operatorname{rank} \mathbf{X}$. If we denote $V_{i}=\mathbf{X}^{T} U_{i} / \sqrt{\lambda}_{i}$, then the SVD of the trajectory matrix can be written as:

$$
\mathbf{X}=\mathbf{X}_{1}+\cdots+\mathbf{X}_{d}
$$

where $\mathbf{X}_{i}=\sqrt{\lambda_{i}} U_{i} V_{i}^{T}$.

The SVD is used to decompose any trajectory matrix $\mathbf{X}$ into two or several orthogonal components. Here, the basic idea is to identify the MECG, FECG and noise components. These components are separated through a successive procedure of configuring $\mathbf{X}, \mathrm{SVD}$, and separation of the most dominant component.

\subsection{Reconstruction}

\section{1st step: Grouping}

The grouping step corresponds to splitting the elementary matrices into several groups and summing the matrices within each group. Let $I=\left\{i_{1}, \ldots, i_{p}\right\}$ be a group of indices $i_{1}, \ldots, i_{p}$. Then matrix $\mathbf{X}_{I}$ corresponding to the group $I$ is defined as $\mathbf{X}_{I}=\mathbf{X}_{i_{1}}+\cdots+\mathbf{X}_{i_{p}}$. The split of the set of indices $J=\{1, \ldots, d\}$ into disjoint subsets $I_{1}, \ldots, I_{m}$ corresponds to the representation

$$
\mathbf{X}=\mathbf{X}_{I_{1}}+\cdots+\mathbf{X}_{I_{m}}
$$

The procedure of choosing the sets $I_{1}, \ldots, I_{m}$ is called the eigentriple grouping.

\section{2nd step: Diagonal averaging}

The purpose of diagonal averaging is to transform a matrix to the form of a Hankel matrix which can be subsequently converted to a time series. If $z_{i j}$ stands for an element of a matrix $\mathbf{Z}$, then the $k$-th term of the resulting series is obtained by averaging $z_{i j}$ over all $i, j$ such that $i+j=k+1$. This procedure is called diagonal averaging, or Hankelization of matrix $\mathbf{Z}$. 


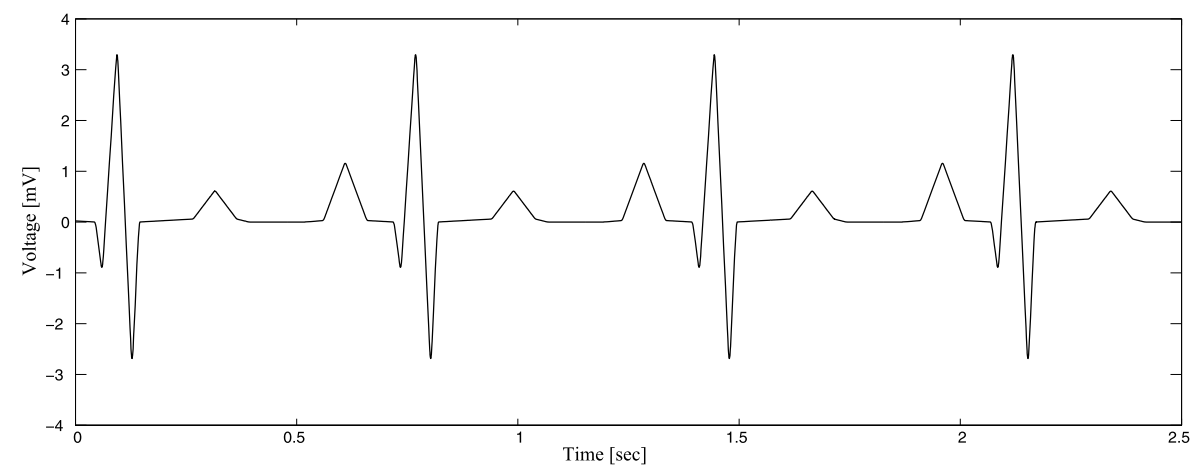

Figure 1. Maternal heartbeat signal.

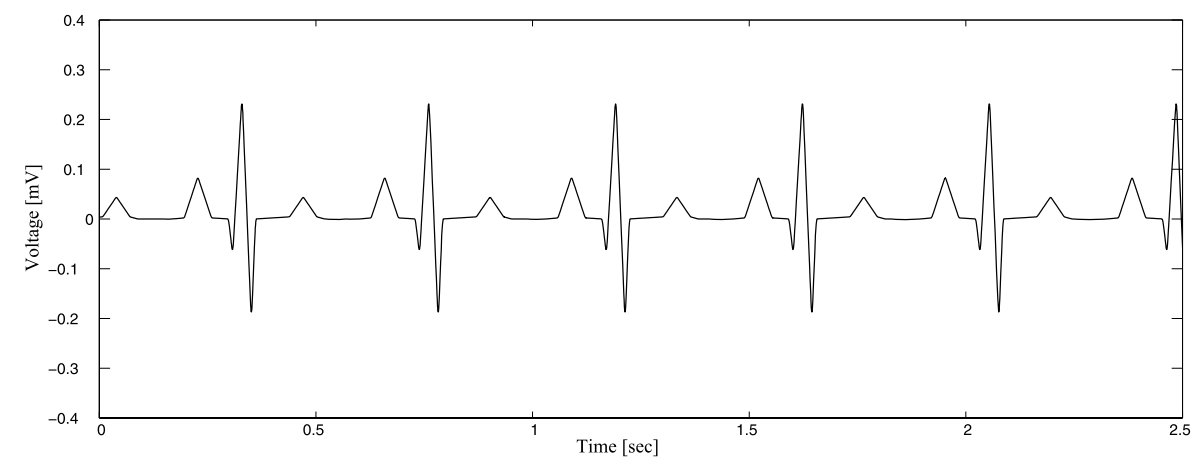

Figure 2. Fetal heartbeat signal.

\subsection{Multivariate singular spectrum analysis}

The use of MSSA for multivariate time series was proposed theoretically in the context of nonlinear dynamics in [37]. There are numerous examples of successful application of MSSA (see, for example, [38] and [26]). Multivariate (or multichannel) SSA is an extension of the standard SSA to the case of multivariate time series.

Assume that we have an $M$-variate time series $y_{j}=$ $\left(y_{j}^{(1)}, \ldots, y_{j}^{(M)}\right)$, where $j=1, \ldots, T$ and let $L$ be window length. Similar to univariate version, we can define the trajectory matrices $\mathbf{X}^{(i)}(i=1, \ldots, M)$ of the one-dimensional time series $\left\{y_{j}^{(i)}\right\}(i=1, \ldots, M)$. The trajectory matrix $\mathbf{X}$ can then be defined as $\mathbf{X}=\left(\mathbf{X}^{(1)} \ldots \mathbf{X}^{(M)}\right)^{T}$.

\section{THE STRUCTURES OF THE FECG AND MECG SIGNALS}

The FECG signal is contained in the composite maternal ECG signal obtained from the abdominal leads. Therefore, the observed signal contains a strong MECG component, a low amplitude FECG component and noise, which may be due to maternal muscle contractions, motion artifacts, etc. Moreover, the periods of both MECG and FECG may vary to a certain extent, and these components are mutu- ally asynchronous. Note that these signals overlap in their frequency bands.

In the following, the proposed MSSA was applied to a simulated signal composed of a mother ECG signal, a fetal ECG signal, and random noise. Both the mother and the fetal simulated ECGs have the same waveform but with different amplitudes and frequencies. Fig. 1 shows a typical simulated MECG signal with a heart rate of approximately 89 beats per minute, and 3.5 millivolts peak voltage.

In general, the fetus has a faster heart rate than the mother. Thus the fundamental frequency of the FECG signal is higher than that of the MECG signal. The fetal heart rates ranging from 120 to 160 beats per minute. The amplitude of the fetal electrocardiogram is also much weaker than that of the maternal electrocardiogram. Fig. 2 shows a typical FECG signal with a heart rate of 139 beats per minute and a peak voltage of 0.25 millivolts.

The mother ECG signal, the fetal ECG signal, and a random noise signal were added together in order to form a mixed signal that mimics the mothers abdominal recording. Fig. 3 shows the composite signal which is a mixture of three different components. Although there is no slowly varying low-frequency component in the series, the composite signal shows the noise level to be considerably high compared to the FECG signal. 


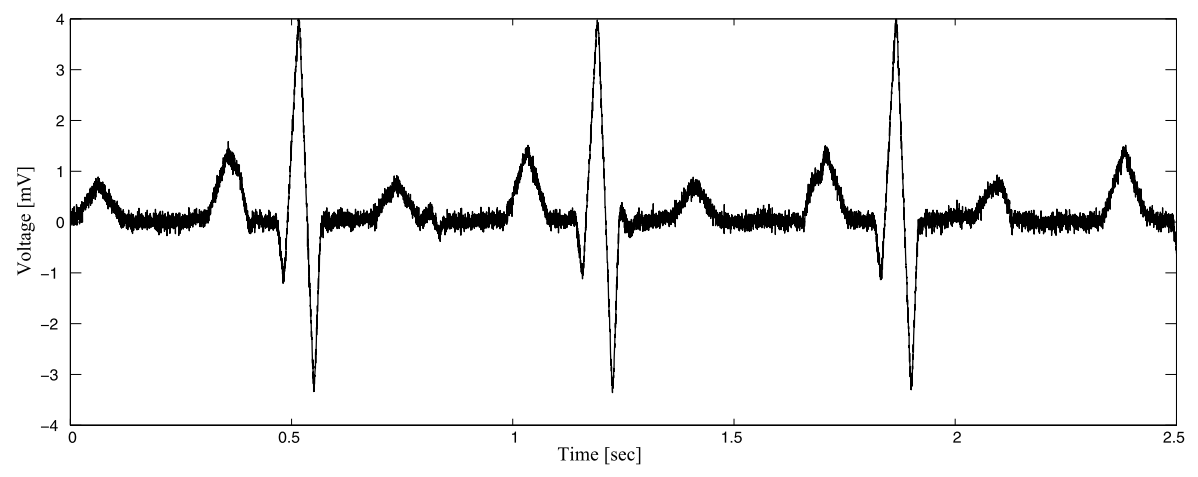

Figure 3. Mixed signal.

\section{DESCRIPTION OF THE PROPOSED ALGORITHM}

Consider a noisy composite signal $Y_{T}=Y_{M}+Y_{F}+Y_{N}$, where the vectors $Y_{M}, Y_{F}$, and $Y_{N}$ represents respectively the mother, the fetal, and the noise components. Fix $L$, the window length, and construct the trajectory matrix $\mathbf{X}=\mathbf{X}_{M}+\mathbf{X}_{F}+\mathbf{X}_{N}$, where $\mathbf{X}_{M}, \mathbf{X}_{F}$ and $\mathbf{X}_{N}$ are trajectory matrices corresponding to maternal, fetal, and the noise components.

In summary, the proposed algorithm consists of two complementary stages. In the first stage, we try to separate the MECG component from the composite signal. The data are first arranged in the form of a trajectory matrix $\mathbf{X}$. We then apply SVD to the trajectory matrix $\mathbf{X}$, and then $\mathbf{X}_{M}$ is separated from $\mathbf{X}$ which contains mother signal, fetal signal, and noise component. We then obtain the matrix $\mathbf{X}_{F N}=\mathbf{X}-\mathbf{X}_{M}$ which contains the FECG component along with noise. The next stage is devoted to extracting the FECG component from $\mathbf{X}_{F N}$. We then apply the SVD step to matrix $\mathbf{X}_{F N}$ to extract the most dominant component that will capture the FECG component.

Let us elaborate on why we first need to extract the MECG in details. There are several reasons. First, the FECG is generated from a very weak heart rate, so the signal is a very low voltage signal. Second, noise from electromyographic activity affects the signal due to its low voltage. Third, another interfering source is the MECG which can be several times higher in its intensity. Moreover, the MECG affects all the electrodes, thoracic and abdominal. There is no place to put an electrode on the mother's skin in order to capture only FECG signal without the mother's signal. Therefore, in all FECG signals, the MECG has higher magnitude. Thus, eliminating the MECG from the recorded signal is very important.

It is worth mentioning that reducing the noise component using classical filtering techniques is not satisfying due to an overlap in spectral content with the FECG. However, removing the noise component step-by-step might lead to an adequate signal to noise ratio. Furthermore, the characteristics of each noise component can be considered as deterministic data to decide which noise reduction method might be useful in order to achieve its removal. Moreover, the noise component sometimes consists of important information of the signal. In this case, using conventional signal processing techniques destroys this information [35].

\section{Preprocessing}

The composite maternal ECG signal may be modulated by a slowly varying low-frequency signal. The preprocessing step includes removal of the slow-varying baseline fluctuation and the power line interference. The baseline fluctuation is, for example, caused by the patient's breathing or movements during recording. These causes have a different frequency from the noise component subspace. For example, the frequency of the baseline wander due to breathing that is in the range of $0.1-0.4 \mathrm{~Hz}$, whilst the artifacts of muscular contractions is characterized by relatively high frequency noise.

We may eliminate the effect of the low-frequency component before the MECG and FECG components are separated. This can be achieved using the Basic univariate SSA. This slow variation can be considered as a trend component in basic SSA. According to the SSA terminology, trend is the slowly varying component of a time series which does not contain oscillatory components. Assume that the time series itself is such a component alone. Empirically in this case, one or more of the leading eigenvectors will be slowly varying as well. We know that eigenvectors have (in general) the same form as the corresponding components of the initial time series. Thus we should find slowly varying eigenvectors. It can be done by considering one-dimensional plots of the eigenvectors.

Let us consider the preprocessing issue using a real MECG signal. Fig. 4 (top) shows the extracted trend (bold line), which is obtained from the first eigentriples, along with MECG signal (thin line). As appears from Fig. 4, the extracted trend clearly follows the main tendency in the se- 

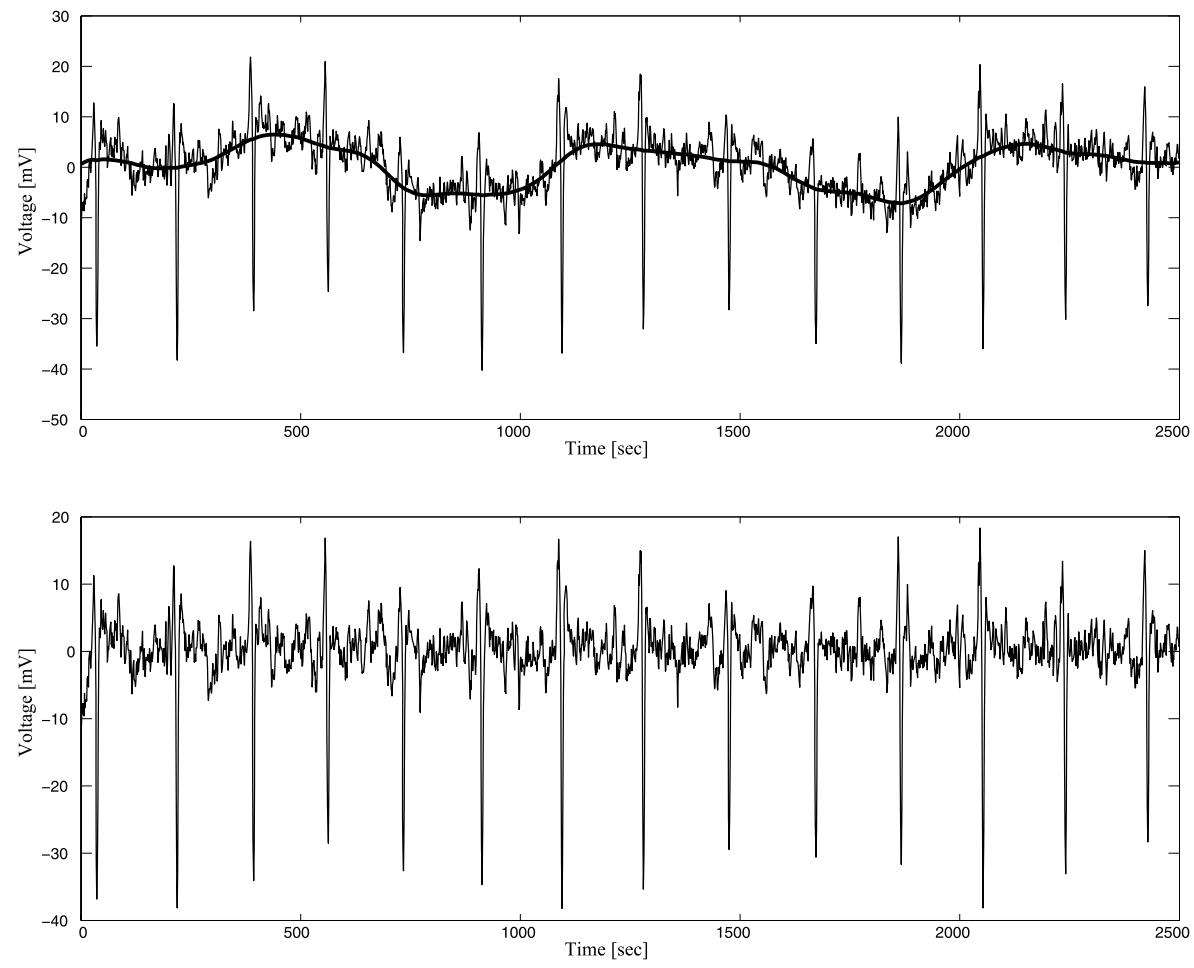

Figure 4. MECG signal and trend reconstruction.

ries. Fig. 4 (bottom) shows the residual signal which is obtained from the remaining eigentriples. Again, Fig. 4 (bottom) clearly shows the same pattern of the MECG signal. In fact, we have obtained the MECG signal from the residual part of the basic SSA after applying it to the initial MECG signal. Therefore, if we are dealing with such a signal, the proposed algorithm will have one more additional step which can be called a preprocessing step.

Note that some other methods have been used for this purpose. For example in [39], each signal has been converted to a zero mean signal by subtracting its mean. It is clear that such a method is not an accurate method, and does not work for all kinds of movements (as in the above example). But here we proposed a more general technique that can be considered for any type of movements.

\section{EMPIRICAL RESULTS}

\subsection{Simulated data}

The electrode pairs are usually chosen according to some specific criteria such as: i) electrode locations on the mother's abdomen (in order to have a better FECG signalto-noise ratio), or ii) the distance (electrode positions are chosen far from the abdomen, e.g. on the thorax such that the MECG is recorded independently and as strongly as possible). For extraction of FECG using MSSA, we always use one signal taken from mother's abdomen, in order to have the baby's signal, and for the other one we have several options; i) placed on the mother's abdomen, ii) placed on the mother's chest, and iii) a mixture of the first and second options. Note that current methods usually use the third option.

To see the importance of the electrode positions for extraction of the FECG signal, we consider all the above conditions for the simulation part. Moreover, to have a better conclusion from the simulated results, we consider the chest signal as a signal that has only the mother's signal (contribution of baby's signal is zero), and in the same way mother's abdomen signal has only baby's signal (contribution of mother's signal is zero). Furthermore, we consider a situation where the baby's and mother's signals overlap since signal overlap is one of the major concerns for several methods used in literature.

Let us first consider the first option; we consider one composite signal (which consists of FECG, MECG and noise components) and the second one from the mother's abdomen (which has only the baby's signal in our cases). As we suggested in the proposed algorithm, we first extract the MECG signal and then the residual (noise component of the first step) shows the pattern of FECG and noise components if any.

The results have been shown in Fig. 5. Fig. 5 (top) shows the noisy composite signal, the middle one represents the extracted MECG signal, and the bottom one shows the noise 

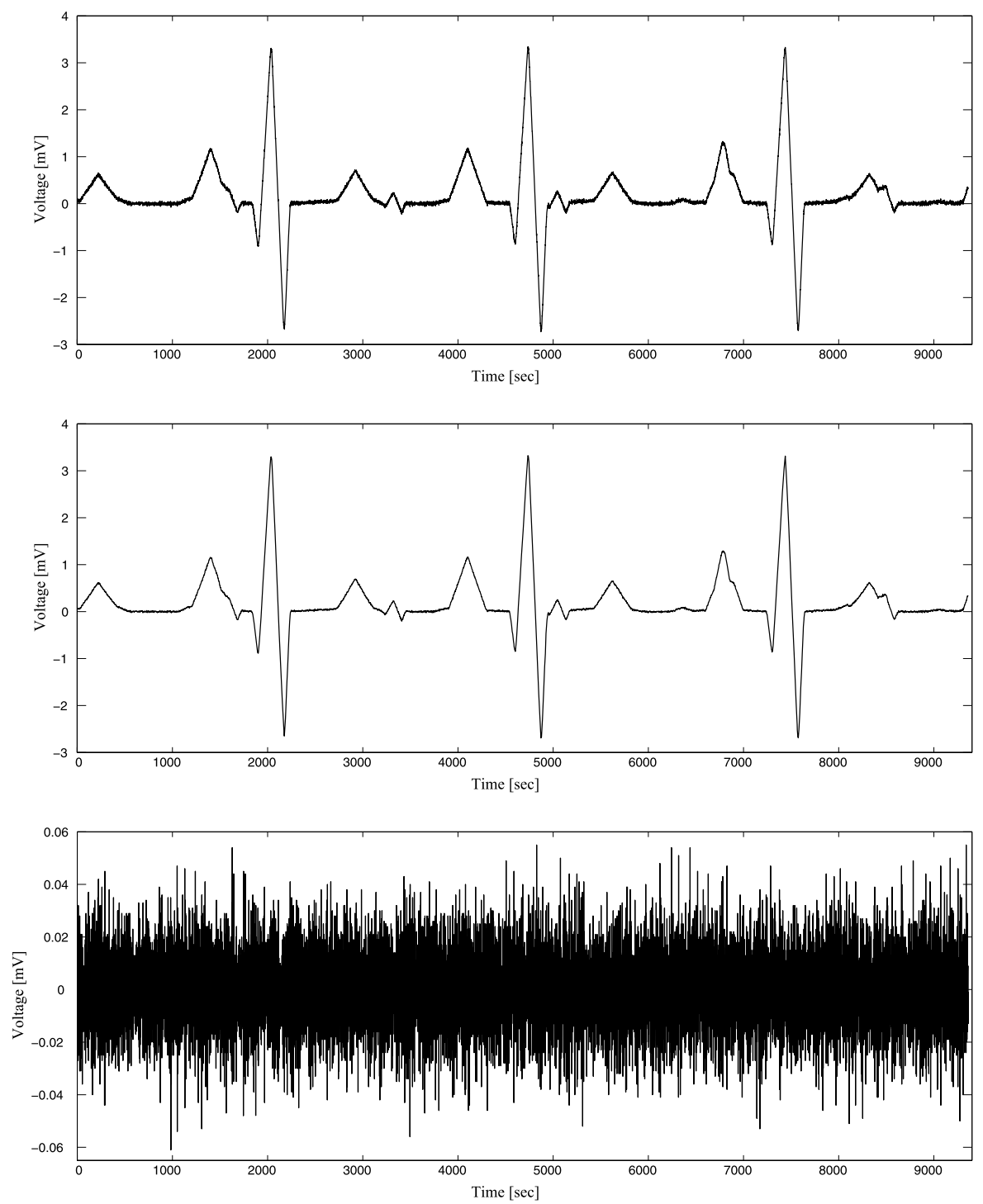

Figure 5. Noisy composite signal (top), extracted MECG (middle) and noise component (bottom).

component. As appears from Fig. 5, having extra information of only the mother's abdomen signal did not help us to extract the FECG signal since there is no evidence of the FECG pattern in noise component. We also added one more signal from the mother's abdomen to see if this extra information helps us to extract the FECG signal. The results indicated that adding one or even two more signals from only mother's abdomen do not provide extra information for extracting the FECG signal. The results are also similar for more than three extra signals. We therefore conclude that adding some extra (simultaneously) abdominal signals will not always result in a better extraction of the FEGG signal. It should be noted that current methods are sensitive to the number of abdominal signals. The more abdominal signals are used in the current methods, the less sensitive the methods are from the particular choice of the electrode pairs [24]. However, this issue does not matter for the proposed algorithm.

Let us now consider the second option. That is, we use the information provided by a composite signal and the extra information obtained from the signals placed on the maternal chest (only mother's signal). Fig. 6 shows the result. The results show the effect of the thoracic signal for extracting the FECG signal. The noise component clearly shows the pattern of the FECG signal. Fig. 6 (top) shows the extracted MECG, the second one (from top) shows the noisy FECG signal (which is, in fact, the residual series or noise component after MECG extraction), the third one represents the extracted FECG signal after reducing noise from the second one, and eventually the last one (bottom) shows the noise component. The results confirm that having only two signals, one from the mother's chest and one from mother's ab- 

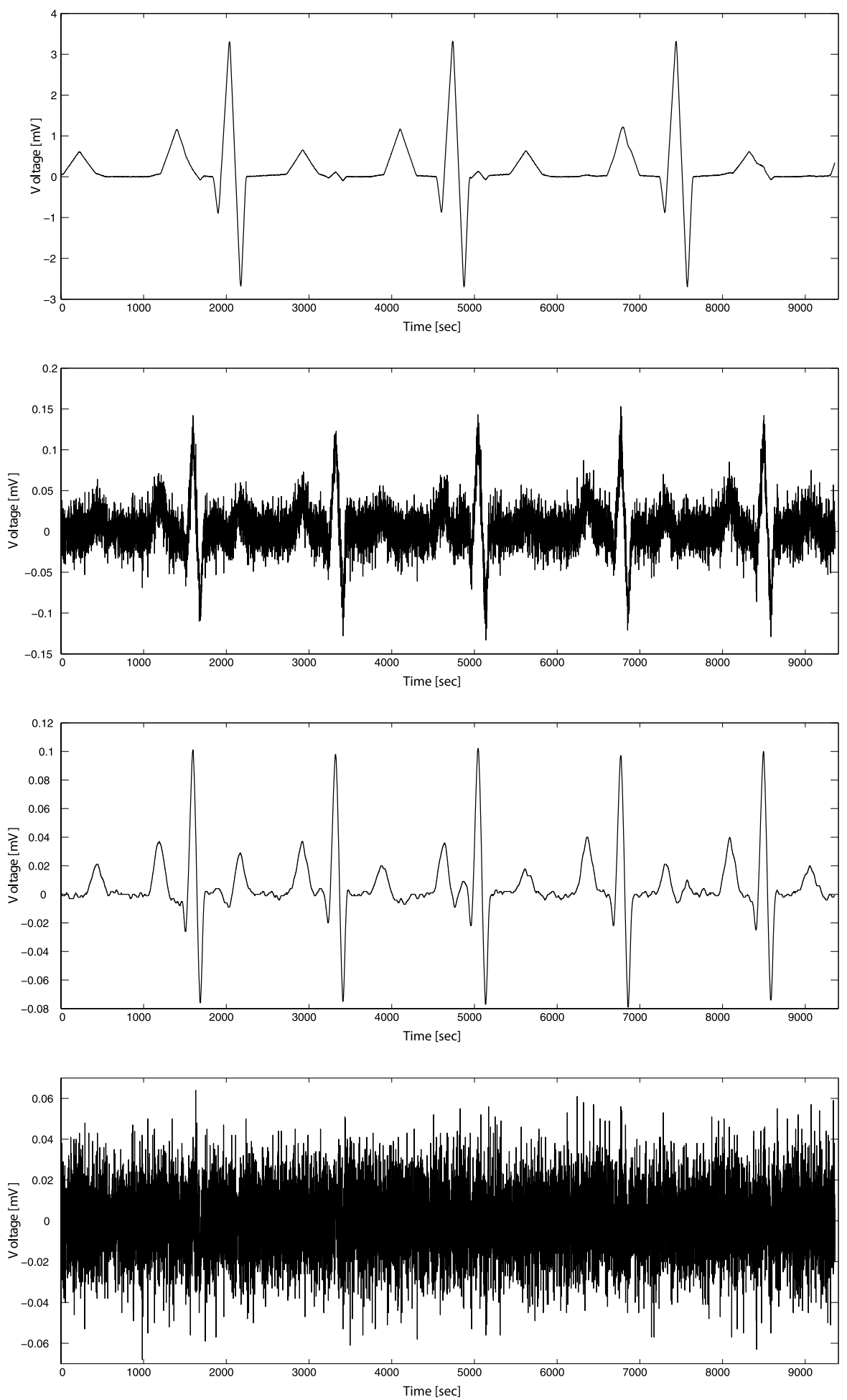

Figure 6. The extracted MECG (top), noisy FECG signal (the second one from top), extracted FECG signal after reducing noise from the second one (the third one from top), and the noise component (bottom). 
domen, enables us to extract both signals MECG and FECG very well. Another important results is that, we are able to correctly capture $\mathrm{P}, \mathrm{Q}$, and $\mathrm{R}$ waves which is an important issue in FECG extraction literature. Thus, by recording one abdominal (composite signal) and one from the chest signal (which is a candidate of the mother's signal) simultaneously, it becomes possible to accurately extract the FECG signal in which the mother ECG bas been suppressed substantially. This motivates adding some more signals from the chest to see if this improves the accuracy of the extraction procedure. It is expected that such information helps since the results in Fig. 6 indicate that only one signal from the chest helps to improve the FECG extraction. Fig. 7 shows the results. Fig. 7 (top) shows the FECG extracted signal with one more additional signal from chest, the middle one with two, and the bottom one with three more additional signals. The results with strong evidence indicate that adding more information from the chest helps to improve the accuracy of the FECG extraction results.

Now consider the last option. That is, we consider one signal from the chest and one signal from the mother's abdomen as extra information simultaneously. Remember that we have a mixture of these signals, and the additional signals are used as extra information. Fig. 8 shows the result. As appears from Fig. 8, there is no discrepancy between the results presented in Fig. 7 and 8 indicating that adding one more signal from the mother's abdomen does not improve the accuracy of the results if we have information of the chest signal. This coincides with the results obtained when we only consider the maternal abdomen signal. In practice, we usually have more information from the mother's abdomen (more sensors are placed on the mother's abdomen) to cover the baby's signal whilst the results obtained here indicate that the attention must also be paid to the signal from the sensor on the chest.

\subsection{Real data}

The results of the simulated series confirm that we are able to extract the FECG signal using two signals; one from maternal abdomen and the other one from the thorax. However, the more signals from thorax, the better FECG extraction results we can obtain. Note that it is difficult to capture the FECG, if the contributions from fetal heart are not sufficient in the abdominal recordings.

Fig. 9 shows the first 5 seconds of a set of six signals recorded in $1 \mathrm{~min}$. The horizontal axis shows the time in seconds and the vertical axes displays amplitude. The value of vertical axes indicates the location of the electrodes. The sampling frequency was $500 \mathrm{~Hz}$. For more information about the data used in this section see [17]. Channels 1-3 show abdominal signals, whilst for the signals in channels 4-6 the electrodes have been placed further away from the fetus, e.g., on the thorax. As appears from Fig. 9, channels 1, 2 and 3 clearly contain weak fetal contributions (due to the large amplitudes of the MECG in the thoracic signals).
Therefore, in these signals the FECG signal is less visible. The results from simulated data confirmed that the residual components of the signals 1-3 show the FECG signals. Therefore, we expect to capture the FECG signals from the first three signals. Fig. 10 shows the results of the first step of the algorithm (the MECG extraction step). Fig. 11 shows the noise component remaining from the first step which will be used for the FECG extraction. As the results indicate, we are able to extract the FECG signal from channels 1-3 as expected, whilst the residual terms of channels 4-6 do not show any pattern of FECG signals (due to the low amplitudes of the FECG signal in the thoracic signals). These results can be considered as another confirmation that the SSA technique can be employed as a powerful tool for signal extraction and separation of a composite signal.

\section{CONCLUSION}

We proposed a method to extract the FECG signal from a noisy composite signal, which is a classical problem in biomedical engineering. The method is based on SSA. The proposed algorithm consists of several complementary stages. First, the maternal ECG is extracted from a noisy composite signal using multivariate SSA. This requires one signal taken from the maternal abdomen, close to the fetal heart, and at least one signal on the thorax. The results clearly confirmed that the SSA technique could be used for extraction the FECG signal and separating noise component.

In fact, here we use multivariate SSA to capture the FECG signal. A considerable advantage of MSSA for this composite signal is that, in MSSA we can capture the dynamic structure between the FECG and MECG signals as a hidden complement information for the extraction of the FECG signal since the SSA technique consists of the elements of multivariate statistics, multivariate geometry and dynamical systems. In the dynamic structure, linear and nonlinear dependencies among the signals are very important concepts in multi-channel analysis that are usually not considered in the current approaches.

The computational aspect of the proposed algorithm can be considered as another advantage since only a few first largest singular values are used.

The proposed algorithm is applicable for extraction of the desired components in any composite signal, where the original signal can be formed in the trajectory matrix $\mathbf{X}$ (consists of two different signals and a noise component). Simplicity and capability of SSA for separation can be considered as other advantages of SSA such that it can be easily adapted to a broad class of biomedical signals.

The implementation of an automated method for maternal and fetal health monitoring and diagnosis during the pregnancy period can be considered as the main outcome of this research in future developments. 

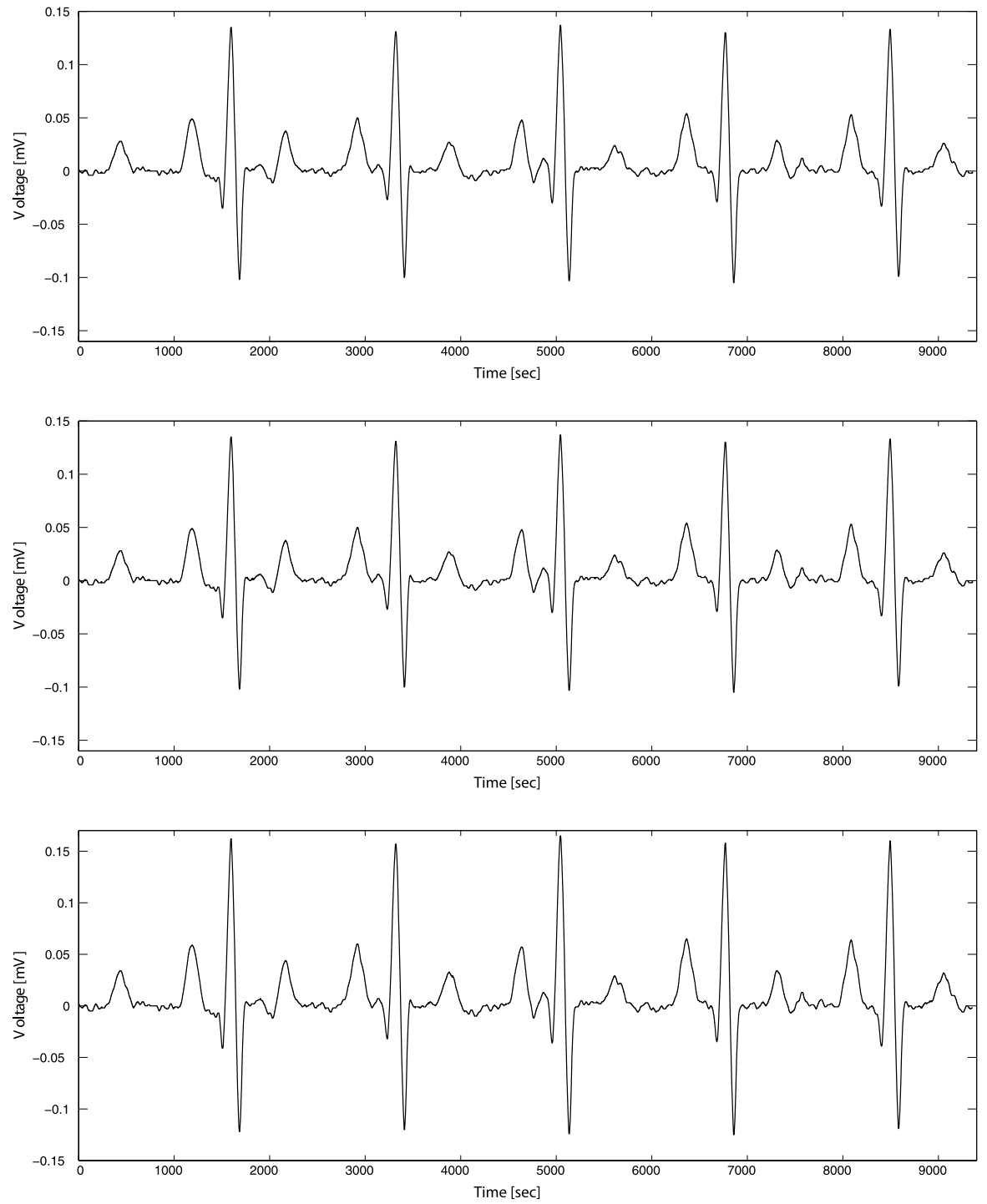

Figure 7. Noise component using one (top), two (middle) and three more mother's abdomen signals (bottom).

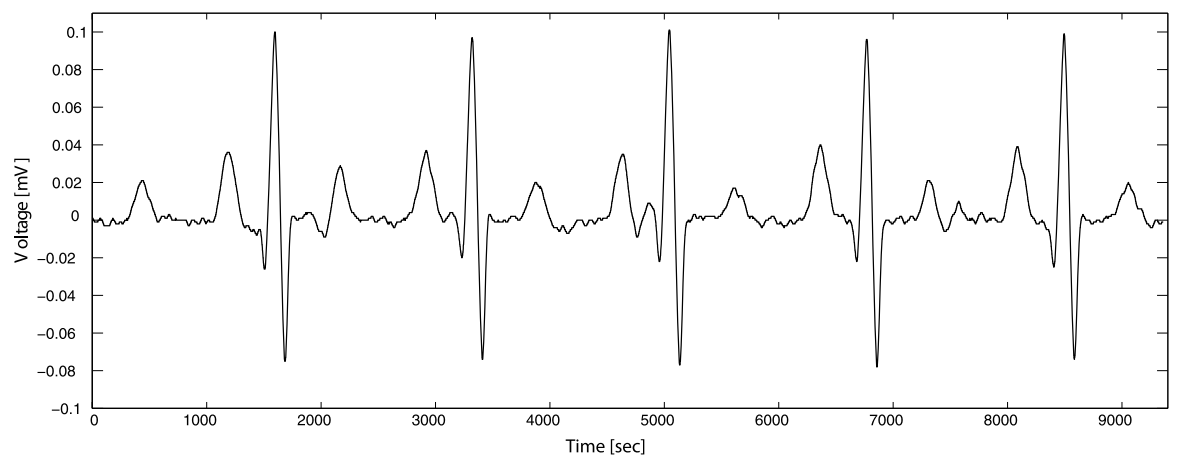

Figure 8. Noise component using one mother's abdomen and one maternal chest signal. 

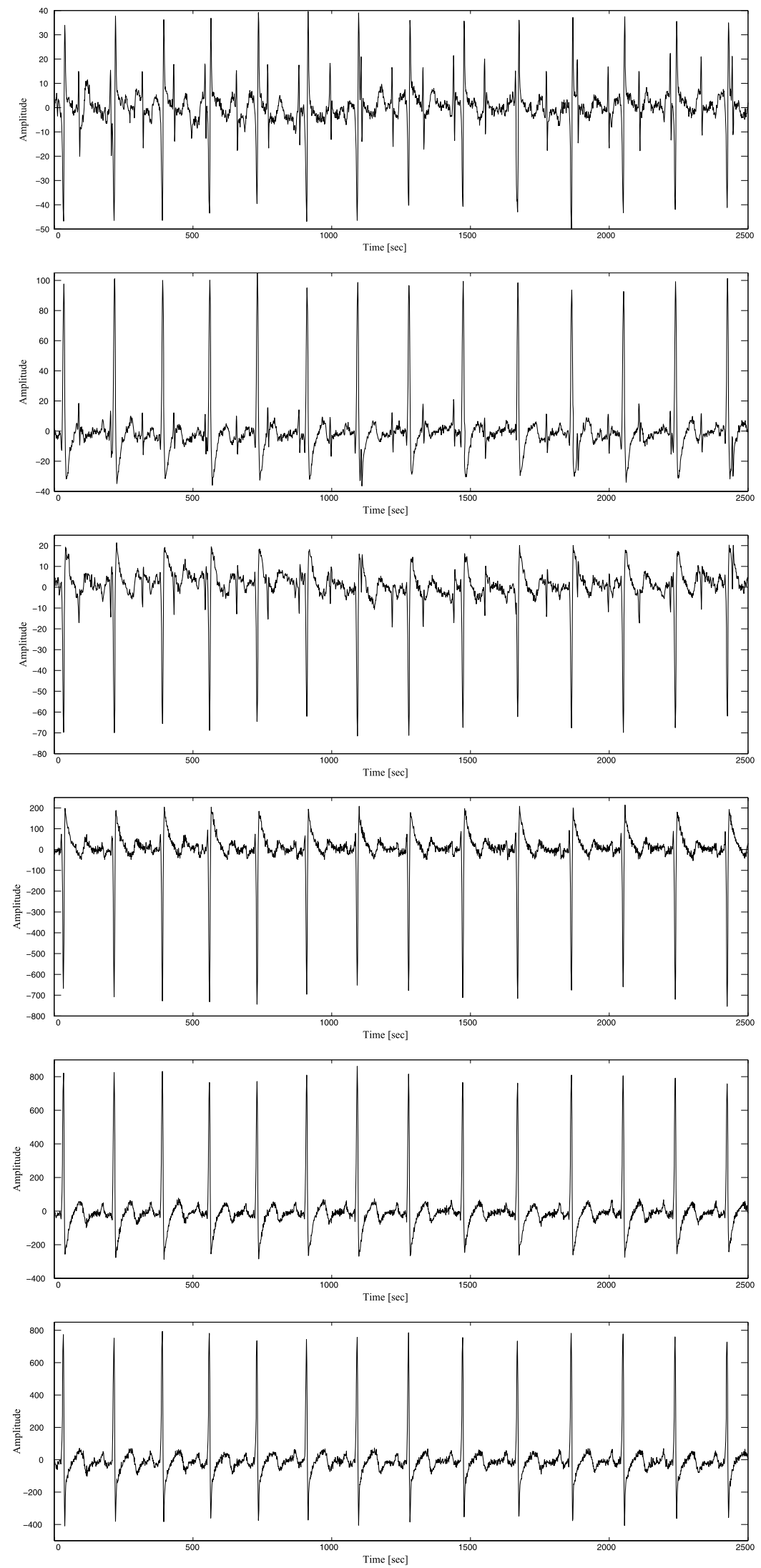

Figure 9. Six-channel composite signals.

408 M. Ghodsi, H. Hassani and S. Sanei 

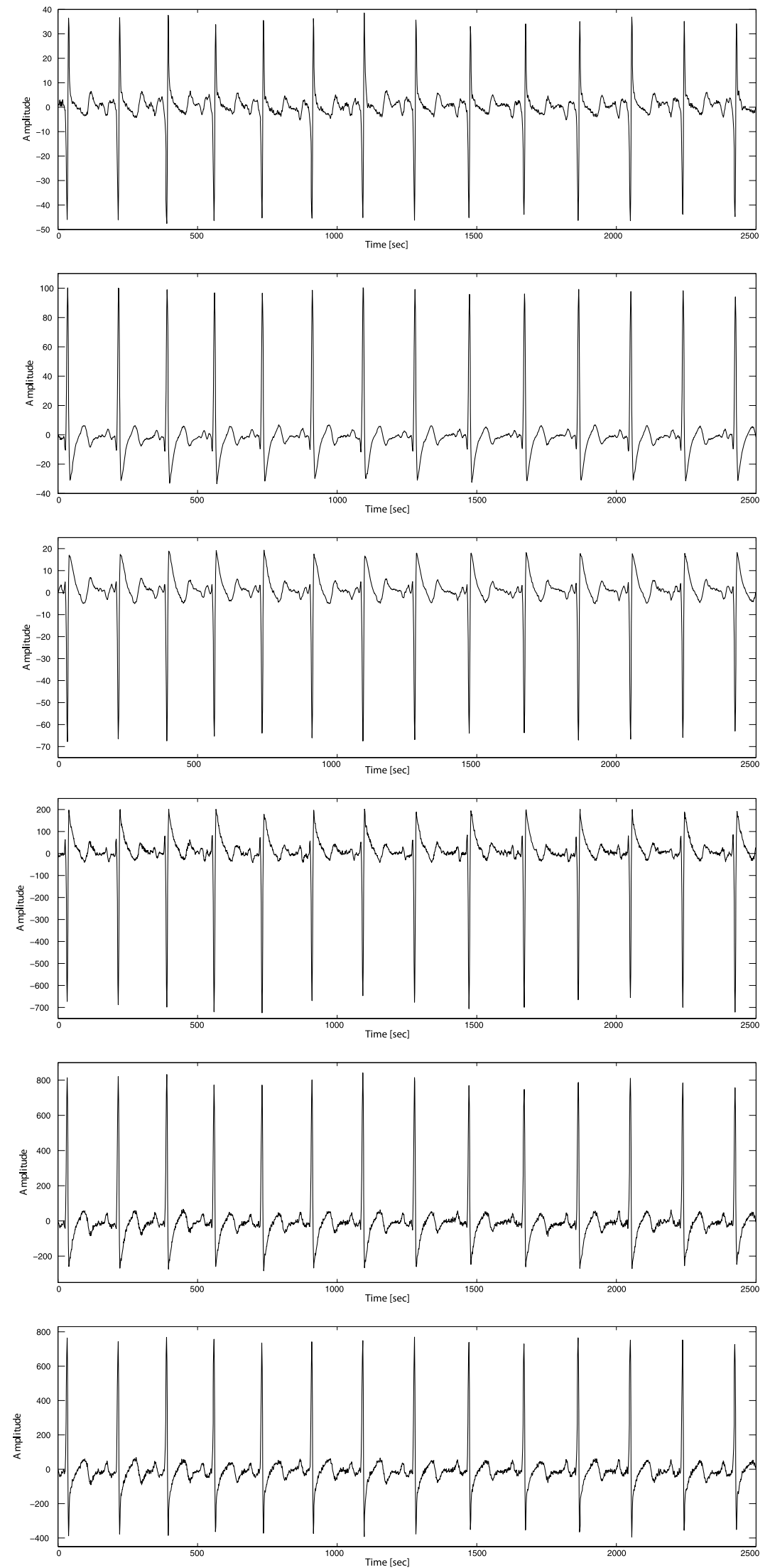

Figure 10. Extracted MECG signal from the real data. 

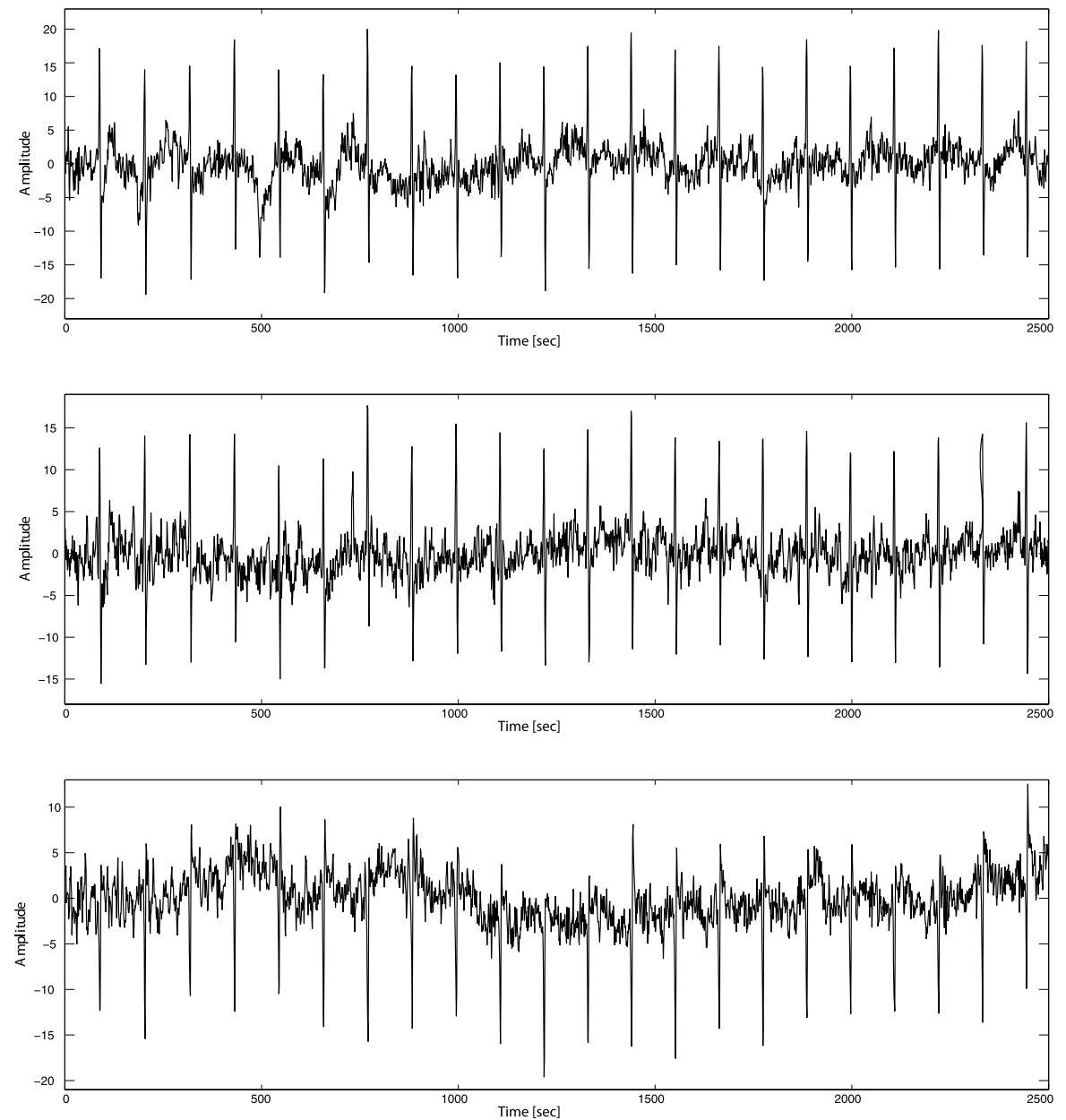

Figure 11. The FECG signal and the noise components extracted from the first step (channels 1-3).

Received 14 January 2010

\section{REFERENCES}

[1] Phelan, J. P. (1989). Tests of few wellbeing ming the few heart rate. Fetal monitoring: Physiology and techniques of antenatal and intrapartum assessment. Ed. I.A.D. Spencer, Castle House Publ. Ltd., Tunbridge Wells.

[2] Camm, A. J., Malik, M., Bigger, J. T., Breithardt, G., Cerutti, S., Cohen, R. J., Coumel, P., Fallen, E. L., Kennedy, H. L., Kleiger, R. E., Lombardi, F., Malliani, A., Moss, A. J., Rottman, J. N., Schmidt, G., Schwartz, P. J. and Singer, D. H. (1996). Guidelines: Heart rate variability; Standards of measurement, physiological interpretation, and clinical use; Task Force of the European Society of Cardiology and the North American Society of Pacing and Electrophysiology. European Heart Journal 17 354-381.

[3] Troeger, C., Schaub, A. F., Bernasconi, P., Hsli, I. and Holzgreve, W. (2003). Spectral Analysis of Fetal Heart Rate Variability in Fetuses with Supraventricular Extrasystoles. Fetal Diagnosis and Therapy 18 284-288.

[4] Friesen, G. M. et AL. (1990). A Comparison of the Noise Sensitivity of Nine QRS Detection Algorithms. IEEE Trans. Biomed. Eng. 37 85-98.

[5] Partha, P. K., Sarbani, P. and Goutam, S. (1997). Fetal ECG
Extraction from Single-Channel Maternal ECG Using Singular Value Decomposition. Med Biol Eng Comput. 44 51-59.

[6] Callaerts, D., De Moor, B., Vandewalle, J., Sansen, W., Vantrappen, G. and Janssens, J. (1990). Comparison of SVD methods to extract the foetal electrocardiogram from cutaneous electrode signals. Med Biol Eng Comput. 28 217-224.

[7] Abboud, S., Alaluf, A., Einav, S. and Sadeh, D. (1992). Realtime abdominal fetal ECG recording using a hardware correlator. Comput Biol Med. 22 325-335

[8] Mooney, D. M., Grooome, L. J., Bentz, L. S. and Wilson, J. D. (1995). Computer algorithm for adaptive extraction of fetal cardiac electrical signal. Proceedings of the ACM symposium on Applied computing 113-117.

[9] Martinez, M., Sofia, E., Calpe, J., Guerrero, J. F. and MaGDAlenA, J. R. (1997). Application of the Adaptive Impulse Correlated Filter for Recovering Fetal Electrocardiogram. Computers in Cardiology 24 9-12.

[10] Longini, R., Reichert, T., Cho, J. and Crowley, J. (1997). Near orthogonal basis functions: A real time fetal ECG technique. IEEE Trans Biomed Eng. 24 29-43.

[11] Richter, M., Schreiber, T. and Kaplan, D. T. (1998). Fetal ECG extraction with nonlinear state space projections. Med Biol Eng Comput. 45133.

[12] Camps, G., Martinez, M. and Sofia, E. (2001). Fetal ECG Extraction using an FIR Neural Network. IEEE, Computers in Cardiology 28 249-252. 
[13] Kam, A. and Cohen, A. (1999). Detection of Fetal ECG with IIR Adaptive Filtering and Genetic Algorithms. IEEE International Conference on Acoustics, Speech, and Signal Processing 4 15-19.

[14] Khandaker, A. K. A. (2000). Fetal QRS Complex Detection from Abdominal ECG: A Fuzzy approach. IEEE Nordic Signal Processing Symposium 275-278.

[15] Barros, A. K. (2002). Extracting the fetal heart rate variability using a frequency tracking algorithm. Neurocomputing 49279 288.

[16] Iванimy, M. I., Ahmed, F., Mohd Ali, M. A. and Zahedi, E. (2003). Real Time Signal Processing for Fetal Heart Rate Monitoring. IEEE Med Biol Eng Comput. 50 258-262.

[17] De Lathauwer, L., De Moor, B. and Vandewalle, J. (2000). Fetal Electrocardiogram Extraction by Blind Source Subspace Separation. IEEE Med Biol Eng Comput. 47 567-572.

[18] Zarzoso, V. and Nandi, A. K. (2001). Noninvasive Fetal Electrocardiogram Extraction: Blind Separation Versus Adaptive Noise Cancellation. Med Biol Eng Comput. 48 12-18.

[19] Khamene, A. and Negahdaripour, S. (2000). A New Method for the Extraction of Fetal ECG from the Composite Abdominal Signal. IEEE Med Biol Eng Comput. 47 507-516.

[20] Mochimaru, F., Fujimoto, F. and Ishikawa, Y. (2002). Detecting the Fetal Electrocardiogram by Wavelet Theory-Based Methods. Progress in Biomedical Research 7 185-193.

[21] Karvounis, E. C., Papaloukas, C., Fotiadis, D. I. and Michalis, L. K. (2004). Fetal Heart Rate Extraction from Composite Maternal ECG Using Complex Continuous Wavelet Transform. Proc. Computers in Cardiology 19-22.

[22] Kotas, M. (2007). Projective filtering of time-aligned beats for foetal ECG extraction. Bulletin of the Polish Academy of Science, Technical Science 55 331-339.

[23] Golyandina, N., Nekrutkin, V. and Zhigluavsky, A. (2001). Analysis of Time Series Structure: SSA and related techniques, Chapman \& Hall/CRC, New York - London. MR1823012

[24] Sameni, R., Clifford, G. D., Jutten, C. and Shamsollahi, M. B. (2007). Multichannel ECG and Noise Modeling: Application to Maternal and Fetal ECG Signals. EURASIP Journal on Advances in Signal Processing 1-14.

[25] Hassani, H., Zhigluavsky, A., Patterson, K. and Soofi, A. (2010). A Comprehensive Causality Test Based on the Singular Spectrum Analysis. Causality in Science, Oxford University press, Forthcoming.

[26] Danilov, D. and Zhigluavsky, A. (1997) (Eds.). Principal Components of Time Series: the 'Caterpillar' method, University of St. Petersburg, St. Petersburg. (In Russian.)

[27] Moskvina, V. G. and Zhigluavsky, A. (2003). An algorithm based on singular spectrum analysis for change-point detection. Communication in Statistics - Simulation and Computation 32 319-352. MR1983338

[28] Hassani, H. (2007). Singular Spectrum Analysis: Methodology and Comparison. Journal of Data Science 5 239-257.

[29] Hassani, H., Heravi, S. and Zhigluavsky, A. (2009). Forecasting European Industrial Production with Singular Spectrum Analysis. Internation Journal of Forecasting 25 103-118.
[30] Patterson, K., Hassani, H., Heravi, S. and Zhigluavsky, A. (2010). Forecasting the final vintage of the industrial production series, Journal of Applied Statistics, Forthcoming.

[31] Hassani, H., Soofi, A. and Zhigluavsky, A. (2009). Predicting Daily Exchange Rate with Singular Spectrum Analysis. Nonlinear Analysis: Real World Applications 11 2023-2034.

[32] Alexandrov, T. H. and Golyandina, N. (2004). The automatic extraction of time series trend and periodical components with the help of the "Caterpillar"-SSA approach. Exponenta Pro 3-4 (In Russian.) 54-61.

[33] www.gistatgroup.com

[34] Hassani, H., Dionisio, A. and Ghodsi, M. (2010). The effect of noise reduction in measuring the linear and nonlinear dependency of financial markets. Nonlinear Analysis: Real World Applications 11 492-502. MR2570568

[35] Ghodsi, M., Hassani, H., Sanei, S. and Hick, Y. (2009). The use of noise information for detecting temporomandibular disorder. Biomedical Signal Processing and Control 4 79-85.

[36] Hassani, H., Zokaei, M., von Rosen, D., Amiri, S. and GHOdsi, M. (2009). Does Noise Reduction Matter for Curve Fitting in Growth Curve Models?. Computer Methods and Program in Biomedicine 96 173-181.

[37] Broomhead, D. S. and King, G. (1986). Extracting qualitative dynamics from experimental data. Physica D 20 217-236. MR0859354

[38] Plaut, G. and Vautard, R. (1994). Spells of low-frequency oscillations and weather regimes in the Northern Hemisphere, $J$ Atmos. Sci. 51 210-236. MR1256578

[39] Algunaidi, M. S. M., Mohd Ali, M. A., Gan, K. B. and ZAHEDI, E. (2009). Fetal Heart Rate Monitoring Based on Adaptive Noise Cancellation and Maternal QRS Removal Window. European Journal of Scientific Research 27 565-575.

\section{Mansoureh Ghodsi}

\section{Cardiff School of Mathematics}

Cardiff University

Cardiff, UK, CF24 4AG

E-mail address: ghodsim@cf . ac.uk

\section{Hossein Hassani \\ Cardiff School of Mathematics \\ Cardiff University \\ Cardiff, UK, CF24 4AG}

E-mail address: hassanih@cf.ac.uk

\section{Saeid Sanei}

Center of Digital Signal Processing, School of Engineering

Cardiff University

Cardiff, UK, CF24 3AA

E-mail address: saneis@cf.ac.uk 